\title{
An integrated approach of predicted miR-34a targets identifies a signature for gastric cancer
}

\author{
TIANTIAN TANG ${ }^{1}$, RONGJIAN $\mathrm{SU}^{2}$, BAOQUAN WANG $^{3}$ and YUNLI ZHANG ${ }^{1}$ \\ ${ }^{1}$ Department of Laboratory Medicine, The First Affiliated Hospital of Liaoning Medical University; \\ ${ }^{2}$ Center of Scientific Experiment, Liaoning Medical University; ${ }^{3}$ Intensive Care Unit, \\ The First Affiliated Hospital of Liaoning Medical University, Jinzhou, Liaoning 121001, P.R. China
}

Received August 15, 2014; April 20, 2015

DOI: $10.3892 / 01.2015 .3266$

\begin{abstract}
RNA-34a (miRNA/miR-34a) functions as a tumor suppressor gene in gastric cancer and may be involved in system-wide regulatory networks. To clarify the expression of all predicted target genes of this miRNA, a comprehensive and systematic analysis of miR-34a-target genes in gastric cancer was conducted in the present study. In the initial analysis, the potential functions, pathways and networks of gastric cancer-associated molecules and miR-34a targets were identified. In the final integrative analysis of gastric cancer-associated miR-34a targets, 30 hub genes were identified using overlap calculations, indicating that miR-34a may be significant in the development and progression of gastric cancer through the Smad signaling pathway, the cell cycle, the mitogen-activated protein kinase signaling pathway, apoptosis, the Notch signaling pathway and other pathways. The present study provides a bioinformatic analysis of miR-34a-targets in gastric cancer, describes numerous target genes and novel coregulatory networks, and may provide an opportunity to identify a critical regulatory network for predicting the molecular mechanisms of miR-34a in the development and progression of gastric cancer.
\end{abstract}

\section{Introduction}

Gastric cancer undergoes genetic and epigenetic alterations during its progression, and is the fourth most common malignancy and the second leading cause of cancer mortality worldwide $(1,2)$. Surgical intervention remains as the preferred treatment for gastric cancer; however, even with intervention, the 5-year survival rate is only $\sim 40 \%$ (3). Cisplatin-based chemotherapy is currently one

Correspondence to: Dr Yunli Zhang, Department of Laboratory Medicine, First Affiliated Hospital of Liaoning Medical University, No. 2, Section 5, Renmin Street, Jinzhou, Liaoning 121001, P.R. China

E-mail: zhangyunligood@gmail.com

Key words: miR-34a, gastric cancer, systematic analysis of the most frequently used therapies. However, numerous patients do not respond to this chemotherapy and must tolerate the associated toxic and adverse effects. Therefore, it is clinically important to distinguish the mechanisms that underlie chemoresistance and the malignant phenotypes of gastric cancer (3). The identification of novel and reliable diagnostic biomarkers and therapeutic strategies is also of the utmost importance (2). Previous studies that sought to identify convincing candidate genes that characterize the heterogeneity of gastric cancer, although far from complete or conclusive, may provide the foundation for systematic analyses of their genetic contributions to this type of tumor, and their regulatory pathways and networks may offer insight into the molecular basis of the pathological or clinical characteristics (1-3).

microRNAs (miRNA/miR) are a class of naturally occurring, small regulatory RNAs that function as negative gene regulators and modulate numerous biological processes, including cellular differentiation, proliferation, apoptosis and metabolism, by targeting varying genes (4). miRNAs have become a major focus in the field of cancer research (5) and the theory that miRNA profiles may reflect the developmental lineage and differentiated state of tumors has been extensively studied in a number of different types of cancer, including gastric cancer (6-8). Notably, miR-34a, which possesses anti-oncogenic activity in certain types of cancer, is downregulated in gastric cancer and cisplatin-resistant cell lines $(9,10)$. A previous study has demonstrated that miR-34a is involved in the sensitivity of gastric cancer to chemotherapies (9). However, the exact molecular mechanism of miR-34a downregulation and its role in gastric cancer development and progression has not been established. Furthermore, it is predicted that a series of factors are involved in the cancer-associated molecular signatures of miR-34a (11). Thus, a comprehensive and systematic analysis of miR-34a-target genes in gastric cancer is of great significance and may provide an opportunity to identify a critical regulatory network for diagnosing and predicting prognosis in gastric cancer.

The present study aimed to systematically analyze the expression of miR-34a predicted target genes associated with tumorigenesis, chemoresistance to cisplatin-based chemotherapy and prognosis in gastric cancer. 


\section{Materials and methods}

Natural language processing (NLP) analysis of gastric cancer. Data selection, extraction and filtering was conducted as previously described (12). The search was performed using the PubMed database (Medline; www.ncbi.nlm.nih.gov/pubmed), attempting to cover all papers published between January 1980 and March 2012, with a combination of the following keywords: 'gastric cancer' AND 'cisplatin' OR 'resistance' OR 'carcinogenesis' OR 'tumorigenesis' OR 'prognosis'; and '1980/01/01' [program delivery assessment tool (PDAT)]: '2012/03/20' (PDAT) (12). All the associated genes and proteins reported in each of the studies were compiled into a list, followed by gene mention tagging using a biomedical named entity recognizer (ABNER; http://pages.cs.wisc.edu/ bsettles/abner/). In addition, the gene symbol in the Entrez gene database of NCBI was considered to be the most common and was therefore used for the study (13). The flow of the NLP analysis was as follows: i) Document searching and formatting; ii) gene mention tagging using ABNER; iii) conjunction resolution; iv) gene name normalization based on the Entrez database; and v) statistical analysis.

Statistical analysis, gene ontology (GO) analysis, pathway analysis and network analysis were also performed as previously described (12).

Statistical analysis. The frequency of the occurrence of each gene was calculated. The higher the frequency of the gene, the greater the likelihood of the association between gastric cancer and that specific gene. The following formulae were used:

$$
\begin{gathered}
p=1-\sum_{i=0}^{k-1} p(i \mid n, m, N) \\
p(i \mid n, m, N)=\frac{n !(N-n) ! m !(N-m) !}{(n-i) ! i !(n-m) !(N-n-m+i) ! N !}
\end{gathered}
$$

$\mathrm{N}$ represents the total number of studies in the literature from the PubMed database; $m$ and $n$ represent the frequency of genes and gastric cancer, respectively, in the literature from the PubMed database; and $\mathrm{k}$ represents the assumption of the actual concomitant occurrence of a gene and a disease. All statistical analyses were performed using GraphPad Prism version 5.0 software (GraphPad Software, Inc., La Jolla, CA, USA). P<0.01 was considered to indicate a statistically significant difference.

Gene ontology. The analysis was conducted using the GSEABase package from the R Project for Statistical Computing platform (www.r-project.org/), and the genes were classified according to biological processes, cellular components and molecular functions.

Pathway analysis. Genes were mapped to the Kyoto Encyclopedia of Genes and Genomes (KEGG) pathway database using GenMAPP software version 2.1 (www.genmapp.org/), and the enrichment P-value was calculated for each pathway (14).

Network analysis. A total of 3 different interaction associations were integrated as previously described (12). Briefly, the pathway data were downloaded from the KEGG database and were then used to analyze the genomic interaction between genes with the KEGGSOAP package from The R Project for Statistical Computing platform (www.bioconductor.org/ packages/2.4/bioc/html/KEGGSOAP.html), including 3 types of associations: Enzyme-enzyme interactions, protein-protein interactions and gene expression interactions (15). The protein-protein interaction data were downloaded from the The MIPS Mammalian Protein-Protein Interaction Database (mips.helmholtz-muenchen.de/proj/ppi/) (16). For interactions that had been previously reported, the co-citation algorithm in the PubMed abstracts was used: The study analyzed whether a gene term and all its term variants co-occurred within the sentences, calculated the frequency of the co-citation gene, and performed a statistical analysis using the same method as described in the NLP analysis. The resulting network was displayed by using the Medusa software (17).

Prediction of miR-34a target genes. The analysis of the miR-34a predicted targets was subsequently determined using a combination of 3 independent software packages as described previously $(12,18)$ : i) PicTar2005 (pictar.mdc-berlin.de/ cgi-bin/PicTar_vertebrate.cgi); ii) miRandaV5 (www.ebi.ac.uk/ enright-srv/microcosm/htdocs/targets/v5/); and iii) TargetScan 5.1 (www.targetscan.org/); GO, pathway and network analyses of miR-34a targets were performed as described in the NLP analysis.

Integrative analysis of miR-34a target genes and NLP results. The overlap of the miR-34a target genes and gastric cancer-associated genes and gene network analysis was subsequently performed.

\section{Results}

NLP analysis of gastric cancer. The initial computerized search identified 22,885 primary studies and a total of 1,183 gastric cancer-associated genes, using the aforementioned search strategies. The 20 most frequently cited genes are listed in Table I. The 1,183 genes were categorized in GO according to biological process, cellular component and molecular function (Fig. 1). Pathway analysis was then performed and indicated that there were 148 pathways available. Among these pathways, the representation in 33 signaling pathways was statistically significant $(\mathrm{P}<0.01$; Table II). It has previously been hypothesized that gene networks reflect the physiological situation as a whole, in addition to the stability of gene regulatory networks and the highly connected hub genes, which are crucial to the stability of the network. Thus, the gene network analysis of the 1,183 identified genes was conducted and is shown in Fig. 2, which presents the relationships between the genes as a whole. A connectivity analysis was also performed. As demonstrated in Fig. 3, the PIK3CA gene has the most interaction gene counts.

Analysis of miR-34a predicted targets. Considering that miRNAs exert biological effects via their numerous targets, the predicted target genes of miR-34a were analyzed using 3 commonly used computational algorithms: TargetScan4.0, PicTar and miRanda. A total of 460 potential unique gene symbols targeted by miR-34a were obtained, and all these genes were categorized by GO analysis (Fig. 4). The gene ontology analysis results for the biological process catergory revealed 
Table I. List of the 20 most frequently cited genes in studies reporting on gastric cancer.

\begin{tabular}{|c|c|c|c|}
\hline Gene & Count & P-value & Description \\
\hline TP53 & 189 & $1.00 \times 10^{-14}$ & Tumor protein p53 \\
\hline$E R B B 2$ & 133 & $1.00 \times 10^{-14}$ & v-erb-b2 erythroblastic leukemia viral oncogene \\
\hline$V E G F A$ & 112 & $1.00 \times 10^{-13}$ & Vascular endothelial growth factor A \\
\hline$B C L 2$ & 106 & $1.00 \times 10^{-13}$ & B-cell CLL/lymphoma 2 \\
\hline$P T G S 2$ & 96 & $1.00 \times 10^{-12}$ & Prostaglandin-endoperoxide synthase 2 (COX-2) \\
\hline$E G F R$ & 94 & $1.00 \times 10^{-12}$ & Epidermal growth factor receptor \\
\hline$J A G 1$ & 57 & $7.54 \times 10^{-9}$ & Jagged 1 (Alagille syndrome) \\
\hline CCND1 & 54 & $1.02 \times 10^{-8}$ & Cyclin D1 \\
\hline TCEAL1 & 53 & $1.00 \times 10^{-11}$ & Transcription elongation factor A (SII)-like 1 \\
\hline MMP9 & 47 & $3.44 \times 10^{-9}$ & Matrix metallopeptidase 9 \\
\hline IL10 & 45 & $1.00 \times 10^{-11}$ & Interleukin 10 \\
\hline$M A P K 8$ & 43 & $1.00 \times 10^{-11}$ & Mitogen-activated protein kinase 8 \\
\hline$D P Y D$ & 40 & $1.00 \times 10^{-11}$ & Dihydropyrimidine dehydrogenase \\
\hline IL6 & 40 & $1.00 \times 10^{-11}$ & Interleukin 6 (interferon, $\beta 2$ ) \\
\hline$C D K N 2 A$ & 39 & $1.00 \times 10^{-11}$ & Cyclin-dependent kinase inhibitor 2A (p16) \\
\hline$T N F$ & 39 & $1.00 \times 10^{-11}$ & Tumor necrosis factor (TNF superfamily, member 2 ) \\
\hline$C D 44$ & 38 & $1.00 \times 10^{-10}$ & CD44 molecule (Indian blood group) \\
\hline MLHI & 35 & $1.00 \times 10^{-10}$ & MutL homolog 1 , colon cancer, nonpolyposis type 2 \\
\hline MAPK3 & 35 & $1.00 \times 10^{-10}$ & Mitogen-activated protein kinase 3 \\
\hline STAT3 & 28 & $1.00 \times 10^{-9}$ & Signal transducer and activator of transcription 3 \\
\hline
\end{tabular}

A

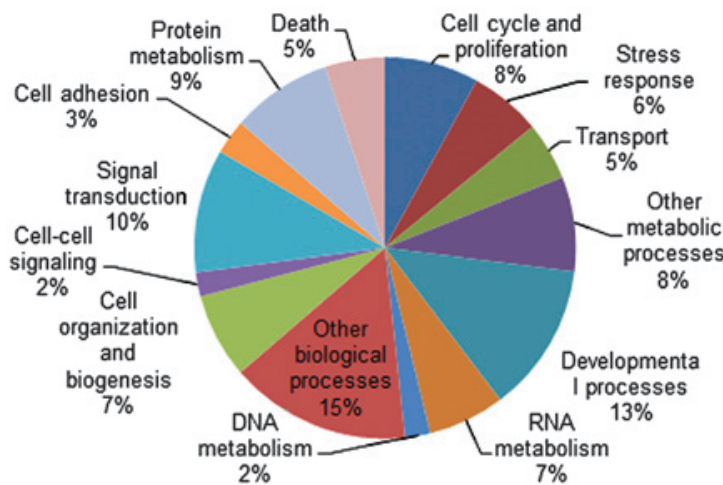

B

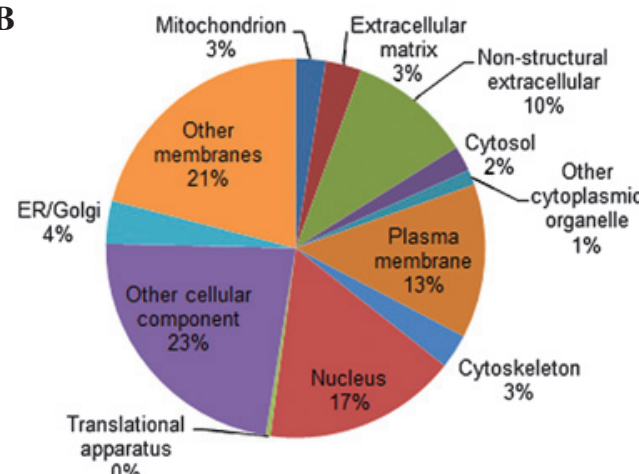

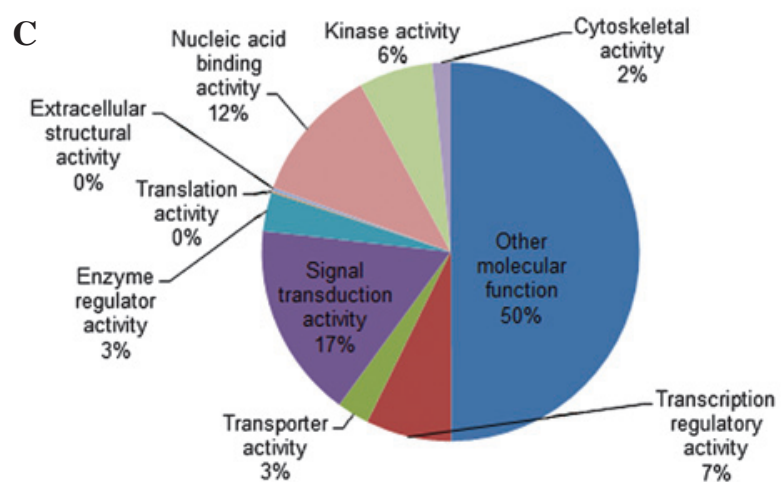

Figure 1. Gene ontology analysis of 1,183 gastric cancer-related genes. (A) Biological process, (B) cellular component and (C) molecular function.

that miR-34a-target genes were predominantly associated with nucleotide metabolic processes, multicellular organism development and cellular component organization. In the pathway analysis, 98 pathways were obtained in the miR-34a targets-pathway. Specific pathways that were identified by the analysis included the PI3K-Akt signaling pathway, the p53 signaling pathway, the notch signaling pathway, adherens junctions, the cell cycle, galactose metabolism and the 


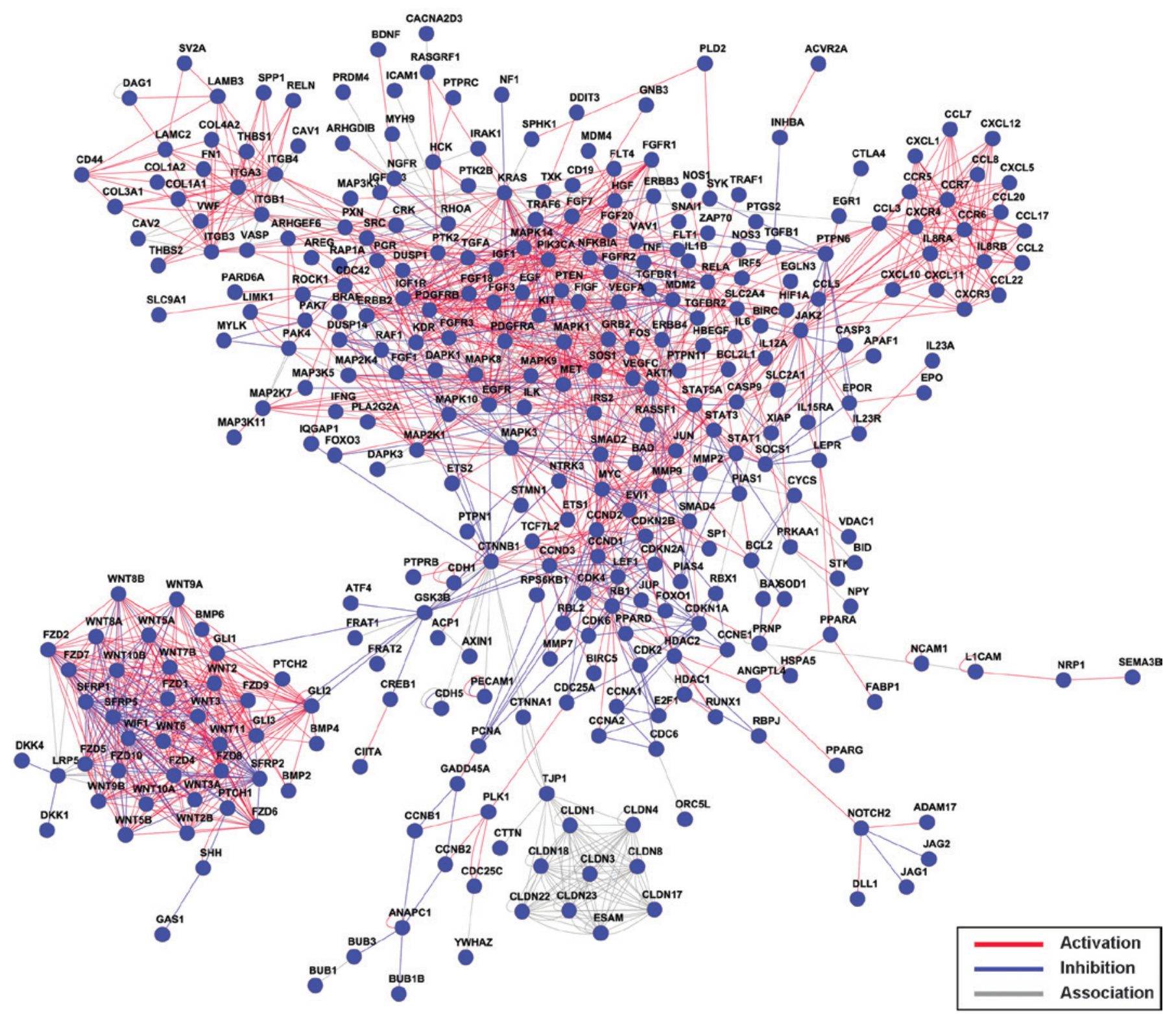

Figure 2. Network analysis of gastric cancer-related genes. Red represents activation, green represents inhibition and gray represents association.

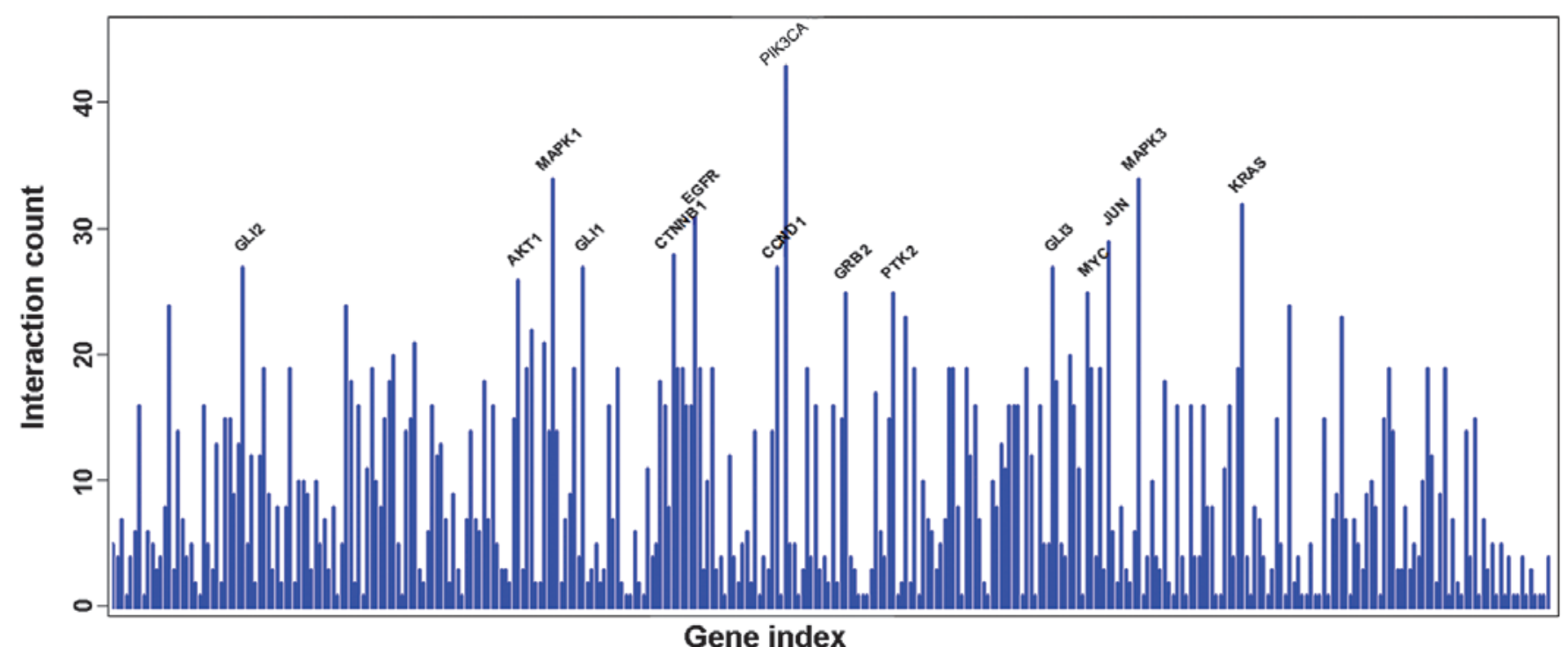

Figure 3. Interaction gene counts for the gastric cancer-related genes. 
Table II. Signaling pathways represented by gastric cancer-associated genes $(\mathrm{P}<0.01)$.

\begin{tabular}{lcc}
\hline Title & Count & P-value \\
\hline p53 signaling pathway & 41 & $2.41 \times 10^{-12}$ \\
Wnt signaling pathway & 56 & $3.25 \times 10^{-12}$ \\
Focal adhesion & 72 & $2.55 \times 10^{-11}$ \\
Cytokine-cytokine receptor interaction & 82 & $3.17 \times 10^{-11}$ \\
ErbB signaling pathway & 35 & $3.02 \times 10^{-10}$ \\
Hedgehog signaling pathway & 27 & $4.02 \times 10^{-10}$ \\
Cell cycle & 40 & $2.04 \times 10^{-12}$ \\
Melanogenesis & 37 & $2.92 \times 10^{-9}$ \\
Neurotrophin signaling pathway & 42 & $6.21 \times 10^{-9}$ \\
T-cell receptor signaling pathway & 37 & $1.75 \times 10^{-8}$ \\
Toll-like receptor signaling pathway & 34 & $1.10 \times 10^{-7}$ \\
Adherens junction & 27 & $5.00 \times 10^{-7}$ \\
Cell adhesion molecules & 39 & $7.98 \times 10^{-7}$ \\
Chemokine signaling pathway & 50 & $1.24 \times 10^{-6}$ \\
Leukocyte transendothelial migration & 34 & $5.12 \times 10^{-6}$ \\
MAPK signaling pathway & 62 & $6.68 \times 10^{-6}$ \\
Apoptosis & 27 & $1.63 \times 10^{-5}$ \\
Hematopoietic cell lineage & 26 & $3.90 \times 10^{-5}$ \\
Jak-STAT signaling pathway & 38 & $1.01 \times 10^{-4}$ \\
Dorso-ventral axis formation & 11 & $1.07 \times 10^{-4}$ \\
Natural killer cell mediated cytotoxicity & 34 & $1.82 \times 10^{-4}$ \\
B-cell receptor signaling pathway & 22 & $2.06 \times 10^{-4}$ \\
TGF-beta signaling pathway & 24 & $2.50 \times 10^{-4}$ \\
FceRI signaling pathway & 22 & $3.81 \times 10^{-4}$ \\
Regulation of actin cytoskeleton & 46 & $4.84 \times 10^{-4}$ \\
VEGF signaling pathway & 21 & $5.73 \times 10^{-4}$ \\
Base excision repair & 12 & $9.71 \times 10^{-4}$ \\
ECM-receptor interaction & 22 & $1.15 \times 10^{-3}$ \\
Adipocytokine signaling pathway & 18 & $2.32 \times 10^{-3}$ \\
mTOR signaling pathway & 15 & $2.48 \times 10^{-3}$ \\
GnRH signaling pathway & 24 & $2.95 \times 10^{-3}$ \\
Antigen processing and presentation & 21 & $4.98 \times 10^{-3}$ \\
Axon guidance & 28 & $5.54 \times 10^{-3}$ \\
\hline & & \\
\hline & & \\
M & &
\end{tabular}

MAPK, mitogen-activated protein kinase; STAT, signal transducers and activators of transcription; TGF, transforming growth factor; VEGF, vascular endothelial growth factor; ECM, extracellular matrix; mTOR, mammalian target of rapamycin; GnRH, gonadotropin-releasing hormone.

HIF-1 signaling pathway. These pathways have already been demonstrated to be involved in the development, progression and chemosensitivity of gastric cancer. Additionally, in the network analysis of the miR-34a predicted targets (Fig. 5), the connectivity of the $C C N D 1$ gene was the highest among the 110 hub genes that were obtained.

Integrative analysis of miR-34a target genes and the NLP results. The overlap between the $460 \mathrm{miR}-34 \mathrm{a}$ target genes and the 1,183 prognosis-associated genes in gastric cancer obtained from the NLP analysis was calculated. A total of

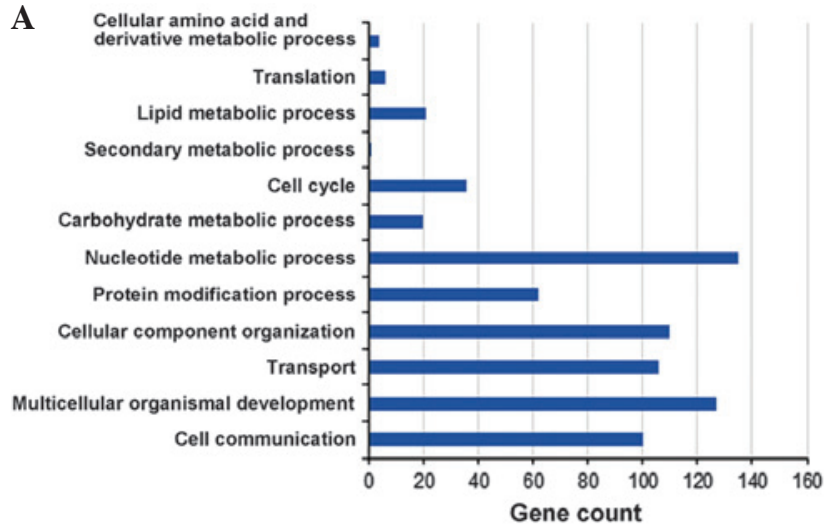

B

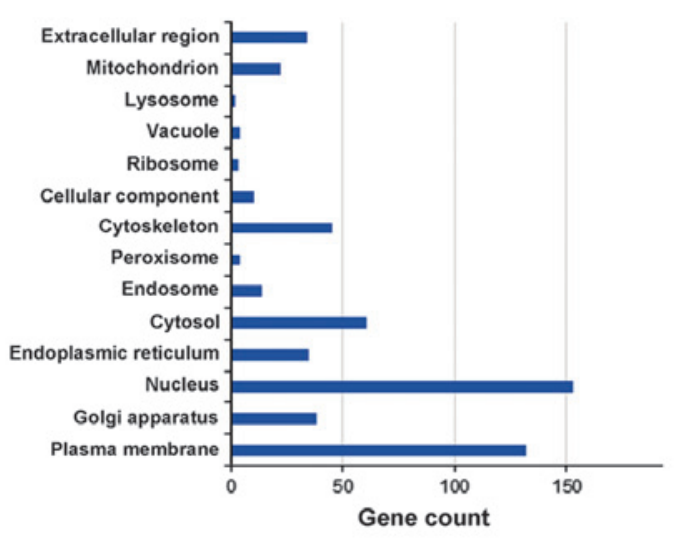

C

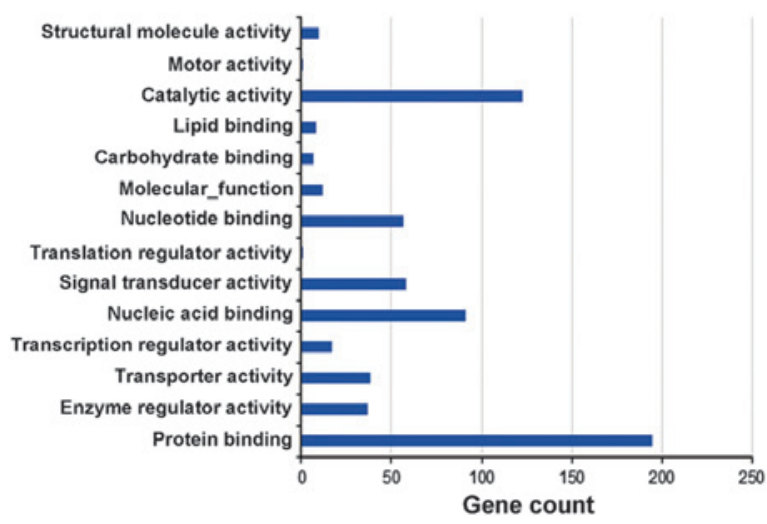

Figure 4. miR-34a-targets genes were categorized in gene ontology analysis. (A) Biological process, (B) cellular component and (C) molecular function.

30 overlap genes that were associated with the development and progression of gastric cancer and that were also potential miR-34a target genes were obtained using this integrative analysis (Table III). A network analysis was also conducted to map the overlapped genes (Fig. 6). From the current results, it appears reasonable to conclude that the $S M A D 4, C C N D 1$, $M A P 2 K 1, B C L 2$ and NOTCH2 hub genes are potential miR-34a target genes and are also essential in the molecular mechanism of gastric cancer. The SMAD4, CCND1, MAP2K1, BCL2 and NOTCH2 genes represent the Smad signaling pathway, the cell cycle, MAPK signaling pathway, apoptosis pathway and the Notch signaling pathway, respectively.

\section{Discussion}

The present study performed a systematic review of a pooled collection of English language studies of gastric cancer-associated 


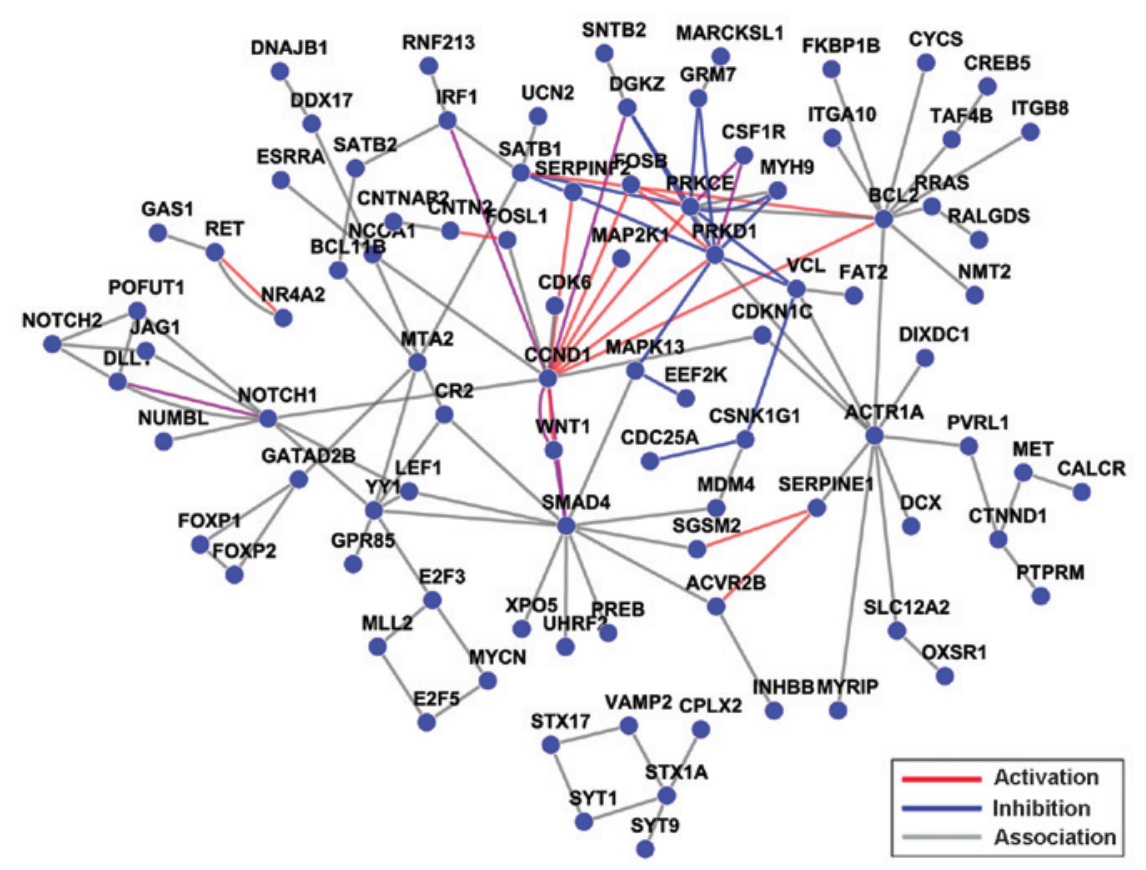

Figure 5. Network analysis of microRNA-34a targets. Red indicates activation, green indicates inhibition and gray indicates association.

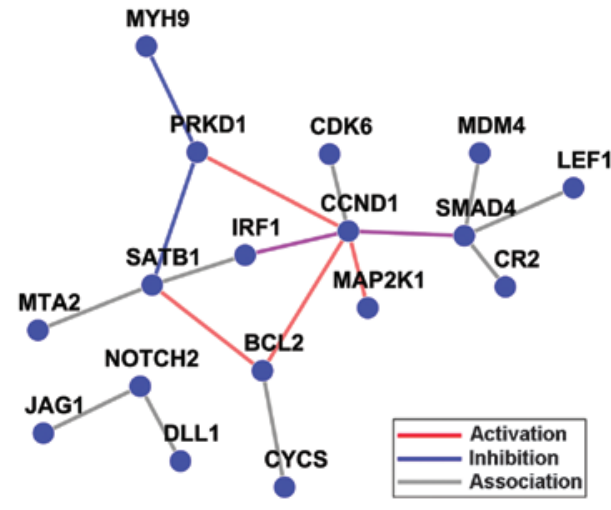

Figure 6. Network analysis of the overlap genes. Red represents activation, green represents inhibition and gray represents association.

molecules. Following classification of the genes into 3 functional groups by GO analysis, gastric cancer-associated networks and pathways were established to identify the key molecules involved. Next, computational methods were used to predict the miR-34a targets, followed by screening for matched gene symbols in the NCBI human sequences and GO, and pathway and network analysis. Finally, in the integrative analysis of gastric cancer-associated miR-34a-targets, hub genes were identified by overlap calculation and the network and pathways of the associated hub genes were further analyzed.

The mechanisms involved in the pathogenesis of gastric cancer are not yet fully clarified. At present, the genes involved in the complex multi-step process of gastric cancer tumor progression, metastasis, relapse and tolerance remain to be fully elucidated. Systematic analysis on the deregulated gene expression, epigenetic or genetic abnormalities may demonstrate their diagnostic potential.

miRNAs have become a major research focus in the field of cancer research (5). A number of miRNAs serve as candidates for clinical biomarkers and have been demonstrated to be useful in characterizing the tumor tissues and reflecting the developmental lineage and differentiated state of cancer $(19,20)$. Previous studies have indicated that miRNAs are involved in the molecular pathogenesis, clinical cancer progression and prognosis of gastric cancer $(8,9,11,21)$. One specific miRNA, miR-34a, has been investigated extensively in various types of tumor, including gastric cancer. Inactivation of miR-34a is a common event during tumorigenesis, and the restoration of miR-34a activity has been indicated to be useful in the prevention of chemotherapy resistance (22). In addition, in gastric mucosa-associated lymphoid tissue lymphoma and diffuse large B-cell lymphoma, reduced expression of miR-34a and increased expression of its target proteins of FOXP1, p53 and BCL2 predict a poor overall survival (11). Moreover, the molecular targets of miR-34a are not limited to those few examples. Furthermore, the present study focused on miR-34a since previous studies have reported malignant activity associated with the downregulation of miR34a and it is often deleted in several cancers, including gastric cancer $(9,23)$. The loss of miR-34a expression has been linked to the resistance to apoptosis induced by the chemotherapeutic p53-activating agents (11). Although the function of miR-34a is relatively well documented, knowledge of miR-34a-targets and miR-34a pathways associated with cancer would provide a more comprehensive understanding of its significance in gastric cancer. One proposed mechanism may be associated with multi-level regulatory control, including tumor suppressor genes, oncogenes and invasion-associated genes. Therefore, for gastric cancer, systematic analysis of malignant behavior-associated miR-34a-targets and their potential molecular mechanisms requires investigation. In the present study, gastric cancer-associated genes and miR-34a target genes were analyzed separately using computational and bioinformatic methods, and then integrated in order to identify the host gene signature of the miR-34a targets. 
Table III. Integrative analysis of miR-34a target genes and the NLP results.

\begin{tabular}{|c|c|c|c|}
\hline Targets & Count & P-value & Gene description \\
\hline$B C L 2$ & 106 & $1.00 \times 10^{-12}$ & B-cell CLL/lymphoma 2 \\
\hline$J A G 1$ & 57 & $7.54 \times 10^{-9}$ & Jagged 1 \\
\hline CCND1 & 54 & $1.02 \times 10^{-8}$ & Cyclin D1 \\
\hline MET & 18 & $1.00 \times 10^{-12}$ & Met proto-oncogene (hepatocyte growth factor receptor) \\
\hline$C Y C S$ & 14 & $1.00 \times 10^{-12}$ & Cytochrome $c$, somatic \\
\hline SERPINE1 & 12 & $1.00 \times 10^{-11}$ & Serpin peptidase inhibitor, clade E, member 1 \\
\hline SMAD4 & 8 & $4.95 \times 10^{-8}$ & SMAD family member 4 \\
\hline$M A P 2 K 1$ & 6 & $1.19 \times 10^{-6}$ & Mitogen-activated protein kinase kinase 1 \\
\hline$A R E G$ & 4 & $5.40 \times 10^{-6}$ & Amphiregulin \\
\hline SATB1 & 4 & $5.49 \times 10^{-7}$ & SATB homeobox 1 \\
\hline$P D C D 4$ & 3 & $5.11 \times 10^{-5}$ & Programmed cell death 4 \\
\hline$C D K 6$ & 3 & $6.78 \times 10^{-4}$ & Cyclin-dependent kinase 6 \\
\hline GAS1 & 2 & $4.45 \times 10^{-4}$ & Growth arrest-specific 1 \\
\hline$I G F B P 3$ & 2 & $1.27 \times 10^{-1}$ & Insulin-like growth factor binding protein 3 \\
\hline IRF1 & 2 & $2.54 \times 10^{-2}$ & Interferon regulatory factor 1 \\
\hline PTPRD & 2 & $7.21 \times 10^{-4}$ & Protein tyrosine phosphatase, receptor type, D \\
\hline$N R 4 A 2$ & 1 & $1.32 \times 10^{-1}$ & Nuclear receptor subfamily 4, group A, member 2 \\
\hline NOTCH2 & 1 & $1.14 \times 10^{-1}$ & Notch homolog 2 \\
\hline PDGFRA & 1 & $2.60 \times 10^{-1}$ & Platelet-derived growth factor receptor, $\alpha$ polypeptide \\
\hline$C D C 25 A$ & 1 & $1.53 \times 10^{-1}$ & Cell division cycle 25 homolog A \\
\hline MTA2 & 1 & $5.66 \times 10^{-2}$ & Metastasis associated 1 family, member 2 \\
\hline $\mathrm{SOX} 4$ & 1 & $5.66 \times 10^{-2}$ & SRY (sex determining region Y)-box 4 \\
\hline МYH9 & 1 & $1.84 \times 10^{-1}$ & Myosin, heavy chain 9, non-muscle \\
\hline DLL1 & 1 & $8.18 \times 10^{-2}$ & ס-like 1 \\
\hline LEF1 & 1 & $1.46 \times 10^{-1}$ & Lymphoid enhancer-binding factor 1 \\
\hline PRKD1 & 1 & $1.50 \times 10^{-1}$ & Protein kinase D1 \\
\hline$J M J D 1 C$ & 1 & $1.86 \times 10^{-2}$ & Jumonji domain-containing $1 \mathrm{C}$ \\
\hline$C R 2$ & 1 & $1.37 \times 10^{-1}$ & Complement component receptor 2 \\
\hline KITLG & 1 & $1.88 \times 10^{-1}$ & KIT ligand \\
\hline MDM4 & 1 & $1.16 \times 10^{-1}$ & Mdm4 p53-binding protein homolog \\
\hline
\end{tabular}

In the NLP analysis, 1,183 genes that were associated with the carcinogenesis, progression and chemoresistance of gastric cancer were identified. The potentially functional classification of the genes was obtained from the GO analysis. The pathway analysis identified 148 pathways and 33 of these were statistically significant, including the p53 signaling pathway, the Wnt signaling pathway, the cell cycle, the MAPK signaling pathway, apoptosis, and the TGF- $\beta$ signaling pathway. A number of previous studies have identified the same pathways to be involved in tumorigenesis, metastasis and chemotherapy resistance $(2,11,24,25)$. In addition, the network and connectivity of those 1,183 genes was constructed in the present study. The highly connected hub genes are crucial to the stability of the network. PIK3CA, with the highest connectivity, had a total of 43 gene connections. A previous study demonstrated that PIK3CA is mutated frequently in a range of human tumors and that its activation is associated with a number of chemotherapeutic agents (26). The results of the present study are consistent with a previous study that analyzed lung cancer-associated genes with NLP and concluded that the gene with the highest connectivity was PIK3CA (12).
In order to obtain the miR-34a target genes in gastric cancer, 3 computational algorithms (miRanda, PicTar, and TargetScan) were used to analyze the predicted targets. From this analysis, 460 unique gene symbols targeted by miR-34a were obtained. These genes were categorized using GO, followed by pathway and network analysis in parallel with the NLP analysis. The results demonstrated that the putative target genes of miR-34a include the tumor-associated genes CCND1, SMAD4, PRKD1, BCL2, NOTCH2 and $S A T B 1$, among others. A total of 98 pathways were obtained in the miR-34a targets pathway analysis, and the PI3K-Akt signaling pathway was identified as the most significant pathway. The 3 genes with the highest connectivity among all 110 hub gene obtained in the miR-34a targets-network analysis were $C C N D 1, S M A D 4$ and BCL2. The CCND1 gene encodes Cyclin D1, a key protein required for $G_{1} / S$ cell cycle transition. Mutations, amplification and overexpression of Cyclin D1 are frequently observed in a number of different types of cancer and may contribute to tumorigenesis (27). SMAD4, which is mutated in a variety of tumors and functions as a tumor suppressor, belongs to the Darwin family of proteins 
that modulate members of the TGF- $\beta$ protein superfamily (28). BCL2 is considered to be an important anti-apoptotic protein and is a member of the BCL2 family of regulator proteins.

In the subsequent integrative analysis of NLP and miR-34a targets, 30 hub genes were obtained. The results indicated that miR-34a is essential in carcinogenesis, progression and the response to chemotherapy in gastric cancer through the Smad signaling pathway, the cell cycle, the MAPK signaling pathway, the apoptosis pathway, the Notch signaling pathway and other pathways. The overlapped targeting hub genes and their pathways may become novel targets for controlling gastric cancer or reversing chemoresistance. Notably, the PIK3CA gene and its pathway, which had the highest connectivity in the NLP analysis, were not involved in the final integrative analysis. This is in agreement with the evidence that PIK3CA was the most significant hub gene in the NLP analysis of lung cancer, but it was not involved in the overlapped analysis with miR-21 (12). The possible explanation for these discrepancies may be that the computational target gene prediction methods have certain limitations in determining actual multifactorial associations.

Collectively, the present study systematically analyzed gastric cancer-associated genes and the putative targets of miR-34a by using a computational and bioinformatics approach. Although additional experiments are required to confirm these results, the systematic integration of miR-34a-targets and their potential modulators provides an efficient approach to discover novel target genes and co-regulatory networks in gastric cancer. Identification of these molecular pathways and networks controlled by miR-34a may provide unique insights into the pathogenesis of gastric cancer.

\section{References}

1. Hartgrink HH, Jansen EP, van Grieken NC and van de Velde CJ: Gastric cancer. Lancet 374: 477-490, 2009.

2. Zhang Z, Miao L, Xin X, Zhang J, Yang S, Miao M, Kong X and Jiao B: Underexpressed CNDP2 participates in gastric cancer growth inhibition through activating the MAPK signaling pathway. Mol Med 20: 17-28, 2014.

3. Correa P: Gastric cancer: Overview. Gastroenterol Clin North Am 42: 211-217, 2013.

4. Bartel DP: MicroRNAs: Genomics, biogenesis, mechanism, and function. Cell 116: 281-297, 2004.

5. Kong YW, Ferland-McCollough D, Jackson TJ and Bushell M microRNAs in cancer management. Lancet Oncol 13: e249-e258, 2012.

6. Aushev VN, Zborovskaya IB, Laktionov KK, et al: Comparisons of microRNA patterns in plasma before and after tumor removal reveal new biomarkers of lung squamous cell carcinoma. PLoS One 8: e78649, 2013.

7. Szabó DR, Luconi M, Szabó PM, et al: Analysis of circulating microRNAs in adrenocortical tumors. Lab Invest 94: 331-339, 2014.
8. Yao Y, Suo AL, Li ZF, et al: MicroRNA profiling of human gastric cancer. Mol Med Rep 2: 963-970, 2009.

9. Cao W, Yang W, Fan R, et al: miR-34a regulates cisplatin-induce gastric cancer cell death by modulating PI3K/AKT/survivin pathway. Tumour Biol 35: 1287-1295, 2014.

10. Li N, Fu H, Tie Y, Hu Z, Kong W, Wu Y and Zheng X: miR-34a inhibits migration and invasion by down-regulation of c-Met expression in human hepatocellular carcinoma cells. Cancer Lett 275: 44-53, 2009.

11. He M, Gao L, Zhang S, Tao L, Wang J, Yang J and Zhu M: Prognostic significance of miR-34a and its target proteins of FOXP1, p53, and BCL2 in gastric MALT lymphoma and DLBCL. Gastric Cancer 17: 431-441.

12. Gao W, Xu J, Liu L, Shen H, Zeng H and Shu Y: A systematic-analysis of predicted miR-21 targets identifies a signature for lung cancer. Biomed Pharmacother 66: 21-28, 2012.

13. Smith L, Tanabe LK, Ando RJ, et al: Overview of BioCreative II gene mention recognition. Genome Biol 9 (Suppl 2): S2, 2008.

14. Dahlquist KD, Salomonis N, Vranizan K, Lawlor SC and Conklin BR: GenMAPP, a new tool for viewing and analyzing microarray data on biological pathways. Nat Genet 31: 19-20, 2002.

15. Ogata H, Goto S, Fujibuchi W and Kanehisa M: Computation with the KEGG pathway database. Biosystems 47: 119-128, 1998.

16. Mewes HW, Albermann K, Heumann K, Liebl S and Pfeiffer F: MIPS: A database for protein sequences, homology data and yeast genome information. Nucleic Acids Res 25: 28-30, 1997.

17. Hooper SD and Bork P: Medusa: A simple tool for interaction graph analysis. Bioinformatics 21: 4432-4433, 2005.

18. Lewis BP, Shih IH, Jones-Rhoades MW, Bartel DP and Burge CB: Prediction of mammalian microRNA targets. Cell 115: 787-798, 2003

19. Volinia S, Calin GA, Liu CG, et al: A microRNA expression signature of human solid tumors defines cancer gene targets. Proc Natl Acad Sci USA 103: 2257-2261, 2006.

20. Rosenfeld N, Aharonov R, Meiri E, et al: MicroRNAs accurately identify cancer tissue origin. Nat Biotechnol 26: 462-469, 2008.

21. Wang F, Sun GP, Zou YF, Hao JQ, Zhong F and Ren WJ: MicroRNAs as promising biomarkers for gastric cancer. Cancer Biomark 11: 259-267, 2012.

22. Hermeking H: The miR-34 family in cancer and apoptosis. Cell Death Differ 17: 193-199, 2010.

23. Cha YH, Kim NH, Park C, Lee I, Kim HS and Yook JI: MiRNA-34 intrinsically links p53 tumor suppressor and Wnt signaling. Cell Cycle 11: 1273-1281, 2012.

24. Kolligs FT, Bommer G and Göke B: Wnt/beta-catenin/tcf signaling: A critical pathway in gastrointestinal tumorigenesis. Digestion 66: 131-144, 2002.

25. Mishra L, Shetty K, Tang Y, Stuart A and Byers SW: The role of TGF-beta and Wnt signaling in gastrointestinal stem cells and cancer. Oncogene 24: 5775-5789, 2005.

26. Endoh H, Yatabe Y, Kosaka T, Kuwano H and Mitsudomi T: PTEN and PIK3CA expression is associated with prolonged survival after gefitinib treatment in EGFR-mutated lung cancer patients. J Thorac Oncol 1: 629-634, 2006.

27. Musgrove EA, Caldon CE, Barraclough J, Stone A and Sutherland RL: Cyclin D as a therapeutic target in cancer. Nat Rev Cancer 11: 558-572, 2011.

28. Inman GJ: Linking Smads and transcriptional activation. Biochem J 386: e1-e3, 2005. 